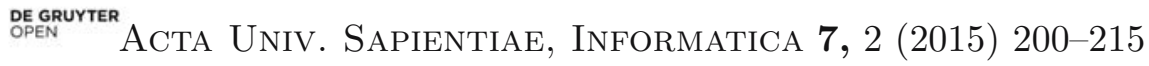

DOI: 10.1515/ausi-2015-0019

\title{
On the scores and degrees in hypertournaments
}

\author{
Shariefuddin PIRZADA \\ University of Kashmir \\ Srinagar, India \\ email: \\ mailto:pirzadasd@kashmiruniversity.ac.in
}

\author{
Rameez RAJA \\ University of Kashmir \\ Srinagar, India \\ email: rameeznaqash@gmail.com
}

\author{
Antal IVÁNYI \\ Eötvös Loránd University \\ Faculty of Informatics \\ Budapest, Hungary \\ email: tony@inf .elte.hu
}

\begin{abstract}
A k-hypertournament $\mathrm{H}=(\mathrm{V}, \mathrm{A})$, where $\mathrm{V}$ is the vertex set and $A$ is an arc set, is a complete $k$-hypergraph with each $k$-edge endowed with an orientation, that is, a linear arrangement of the vertices contained in the edge. In a k-hypertournament, the score $s_{i}$ (losing score $r_{i}$ ) of a vertex is the number of edges containing $v_{i}$ in which $v_{i}$ is not the last element(in which $v_{i}$ is the last element) and the total score of a vertex $v_{i}$ is $t_{i}=s_{i}-r_{i}$. For $v \in V$ we denote $d_{H}^{+}=\sum_{a \in H} \rho(v, a)$ (or simply $d^{+}(v)$ ) the degree of a vertex where, $\rho(v, a)$ is $k-i$ if $v \in a \in A$ and $v$ is the ith entry in $a$, otherwise zero. In this paper, we obtain necessary and sufficient conditions for a k-hypertournament to be degree regular. We use the inequalities of Holder and Chebyshev from mathematical analysis to study the score and degree structure of the k-hypertournaments.
\end{abstract}

Computing Classification System 1998: G.2.2

Mathematics Subject Classification 2010: 05C30, 05C50

Key words and phrases: tournaments, hypertournaments, score, losing score, degree 


\section{Introduction}

Hypertournaments which are the generalizations of tournaments, have been studied by number of authors like R. Assous [1], Barbut and Bialostocki [2], P. Frank [4] and Gutin and Yeo [5]. These authors raise the problem of extending the most important results on tournaments to hypertournaments. G. Zhou et al. [26] extended the concept of scores in tournaments to that of scores and losing scores in hypertournaments, and derived various results $[6,14,15,16$, $17,18,19,21,10,11,12,13,22,23,25]$.

Given two non-negative integers $n$ and $k$ with $n \geq k>1$, a k-hypertournament $H$ on $n$ vertices is a pair $(V, A)$, where $V$ is the set of vertices with $|\mathrm{V}|=\mathrm{n}$, and $\mathrm{A}$ is the set of $\mathrm{k}$-tuples of vertices called arcs, such that for any $k$-subset $S$ of $\mathrm{V}, \mathrm{A}$ contains exactly one of the $\mathrm{k}$ ! $\mathrm{k}$ tuples whose entries belong to $S$.

Zhou et al. [26] extended the concept of scores in tournaments to that of scores and losing scores in hypertournaments, and derived a result analogous to Landau's theorem [9] on tournaments. The score $s\left(v_{i}\right)$ or $s_{i}$ of a vertex $v_{i}$ is the number of arcs containing $v_{i}$ in which $v_{i}$ is not the last element and the losing score $r\left(v_{i}\right)$ or $r_{i}$ of a vertex $v_{i}$ is the number of arcs containing $v_{i}$ in which $v_{i}$ is the last element. The score sequence (losing score sequence) is formed by listing the scores(losing scores) in non-decreasing order. A k-hypertournament is said to be regular if the scores of each vertex (equivalently the losing scores) are same.

Let $\mathrm{H}$ be a k-hypertournament and let $\nu \in \mathrm{V}$ be any vertex and $\mathrm{a}=$ $\left(v_{1}, v_{2}, \ldots, v_{n}\right) \in A$ be an arc of $\mathrm{H}$. We denote by $\mathrm{d}_{\mathrm{H}}^{+}=\sum_{\mathrm{a} \in \mathrm{H}} \rho(v, \mathrm{a})$ (or simply $\left.\mathrm{d}^{+}(v)\right)$ the degree of a vertex $v \in \mathrm{V}$ where,

$$
\rho(v, a)= \begin{cases}k-i, & \text { if } v \in a \text { and } v \text { is the } i^{\text {th }} \text { entry of a, } \\ 0, & \text { if } v \notin a .\end{cases}
$$

A hypertournament is said to be degree regular if all the vertices have the same degree. The degree sequence of a k-hypertournament in non-decreasing order is a sequence of non-negative integers $\left[d_{1}, d_{2}, \ldots, d_{n}\right]$, where each $d_{i}$ is the degree of some vertex in $\mathrm{V}$.

The following characterizations of losing score sequences of k-hypertournaments can be found in [26], and a new short proof is given by Pirzada et al. in $[20]$. 
Theorem 1 Given two non-negative integers $\mathrm{n}$ and $\mathrm{k}$ with $\mathrm{n} \geq \mathrm{k}>1$, a non-decreasing sequence $\mathrm{R}=\left[\mathrm{r}_{1}, \mathrm{r}_{2}, \ldots, \mathrm{r}_{\mathrm{n}}\right]$ of non-negative integers is a losing score sequence of some $\mathrm{k}$-hypertournament if and only if for each $1 \leq \mathrm{j} \leq \mathrm{n}$,

$$
\sum_{i=1}^{j} r_{i} \geq\left(\begin{array}{l}
j \\
k
\end{array}\right)
$$

with equality when $\mathrm{j}=\mathrm{n}$.

Theorem 2 Given two integers $\mathrm{n}$ and $\mathrm{k}$ with $\mathrm{n} \geq \mathrm{k}>1$, a non-decreasing sequence $\mathrm{S}=\left[\mathrm{s}_{1}, \mathrm{~s}_{2}, \ldots, \mathrm{s}_{\mathrm{n}}\right]$ of non-negative integers is a score sequence of some $\mathrm{k}$-hypertournament if and only if for each $1 \leq \mathrm{j} \leq \mathrm{n}$,

$$
\sum_{i=1}^{j} s_{i} \geq j\left(\begin{array}{l}
n-1 \\
k-1
\end{array}\right)+\left(\begin{array}{c}
n-j \\
k
\end{array}\right)-\left(\begin{array}{l}
n \\
k
\end{array}\right)
$$

with equality when $\mathbf{j}=\mathfrak{n}$.

Koh and Ree [7] defined the k-hypertournament matrix $M=M(H)$ associated with a k-hypertournament $H=(V, A)$ as the incidence matrix $M=\left[m_{i j}\right]$ of size $n \times\left(\begin{array}{l}n \\ k\end{array}\right)$ of $H$, where for $1 \leq i \leq n$ and $1 \leq j \leq\left(\begin{array}{l}n \\ k\end{array}\right), m_{i j}$ is given by

$m_{i j}= \begin{cases}1, & \text { if } v_{i} \in e_{j} \text { and } v_{i} \text { is not the last element of } e_{j}, \\ -1, & \text { if } v_{i} \in e_{j} \text { and } v_{i} \text { is the last element of } e_{j}, \\ 0, & \text { if } v_{i} \notin e_{j} .\end{cases}$

Since $s_{i}+r_{i}=\left(\begin{array}{l}n-1 \\ k-1\end{array}\right)$ for each $i$, then clearly a given sequence $s_{1} \geq s_{2} \geq \ldots \geq$ $s_{n} \geq 0$ is a score sequence of a k-hypertournament.

Theorem 3 A non-increasing sequence of non-negative integers $s_{1} \geq s_{2} \geq$ $\ldots \geq s_{\mathfrak{n}} \geq 0$ is a score sequence of a $\mathrm{k}$-hypertournament $\mathrm{H}$ if and only if

$$
\sum_{i=1}^{l} s_{i} \leq l\left(\begin{array}{l}
n-1 \\
k-1
\end{array}\right)-l\left(\begin{array}{l}
l \\
k
\end{array}\right)
$$

for $\mathrm{l}=\{1,2, \ldots, \mathrm{n}\}$ with equality when $\mathrm{l}=\mathrm{n}$. 
Theorem 4 Sequences $0 \leq s_{1} \leq s_{2} \leq \ldots \leq s_{n} \leq$ and $r_{1} \geq r_{2} \geq \ldots \geq r_{n} \geq 0$ are the score and losing score sequence of a $\mathrm{k}$-hypertournament $\mathrm{H}$ if and only if they satisfy $s_{i}+r_{i}=\left(\begin{array}{l}n-1 \\ k-1\end{array}\right)$ for all $i=\{1,2, \ldots, n\}$,

$$
\sum_{i=1}^{l} s_{i} \geq l\left(\begin{array}{l}
n-1 \\
k-1
\end{array}\right)+l\left(\begin{array}{c}
n-l \\
k
\end{array}\right)-\left(\begin{array}{l}
n \\
k
\end{array}\right) \text {, }
$$

and

$$
\sum_{i=1}^{l} r_{i} \leq\left(\begin{array}{l}
n \\
k
\end{array}\right)-\left(\begin{array}{c}
n-l \\
k
\end{array}\right),
$$

for $\mathrm{l}=\{1,2, \ldots, \mathrm{n}\}$ with equality when $\mathrm{l}=\mathrm{n}$.

The following result [7] gives the condition for a sequence to be the total score sequence of a k-hypertournament matrix.

Theorem 5 A non-increasing sequence of integers $\mathrm{T}=\left[\mathrm{t}_{1}, \mathrm{t}_{2}, \ldots, \mathrm{t}_{\mathrm{n}}\right]$ is the total score sequence of a $\mathrm{k}$-hypertournament matrix $M$ on $\mathrm{n}$ vertices if and only if $\mathrm{t}_{\mathrm{i}}$ has the same parity as that of $\left(\begin{array}{c}n-1 \\ k-1\end{array}\right)$, for all $i=\{1,2, \ldots, n\}$ and

$$
\sum_{i=1}^{l} t_{i} \leq l\left(\begin{array}{l}
n-1 \\
k-1
\end{array}\right)-2\left(\begin{array}{l}
l \\
k
\end{array}\right),
$$

for $\mathrm{l}=\{1,2, \ldots, \mathrm{n}\}$ with equality when $\mathrm{l}=\mathrm{n}$.

Khan, Pirzada and Kayibi [8] applied the inequalities from mathematical analysis like Holder's, Minkowski's and Mahler's inequalities to the powers of scores and losing scores of k-hypertournaments and obtained the following results. The result below [8] gives a lower bound on $\sum_{i=i}^{j} r_{i}^{g}$, where $1<g<\infty$ is a real number.

Theorem 6 [8]. Let $\mathrm{n}$ and $\mathrm{k}$ be two non-negative integers with $\mathrm{n} \geq \mathrm{k}>1$. If $\left[\mathrm{r}_{1}, \mathrm{r}_{2}, \ldots, \mathrm{r}_{\mathrm{n}}\right]$ is a losing score sequence of a $\mathrm{k}$-hypertournament, then for $1<\mathrm{g}<\infty$

$$
\sum_{i=i}^{j} r_{i}^{g} \geq \frac{j}{k^{g}}\left(\begin{array}{l}
j-1 \\
k-1
\end{array}\right)^{g},
$$

where $1 \leq \mathfrak{j}<\mathrm{n}$. In particular, for $\mathbf{j}=\mathrm{n}$

$$
\sum_{i=i}^{n} r_{i}^{g} \geq \frac{n}{k^{g}}\left(\begin{array}{l}
n-1 \\
k-1
\end{array}\right)^{g},
$$


with equality in (3) holds if and only if the hypertournament is regular.

The next result [8] gives an upper bound for the inner product of score and losing score vectors in $\mathrm{R}^{\mathfrak{n}}$. The bound given in Theorem 7 is best possible in the sense that it is realized by regular hypertournaments. It should also be noted that Theorem 7 does not depend on the order of $s_{i}$ and $r_{i}$, and holds for any arbitrary ordering of scores and losing scores.

Theorem 7 Let $\mathrm{n}$ and $\mathrm{k}$ be two non-negative integers with $\mathrm{n} \geq \mathrm{k}>1$. If $\mathrm{S}=\left[\mathrm{s}_{1}, \mathrm{~s}_{2}, \ldots, \mathrm{s}_{\mathrm{n}}\right]$ and $\mathrm{R}=\left[\mathrm{r}_{1}, \mathrm{r}_{2}, \ldots, \mathrm{r}_{\mathrm{n}}\right]$ are respectively the score and losing score sequence of a k-hypertournament, then

$$
\langle S, R\rangle=\sum_{i=1}^{n} s_{i} r_{i} \leq \frac{k-1}{k}\left(\begin{array}{l}
n \\
k
\end{array}\right)\left(\begin{array}{l}
n-1 \\
k-1
\end{array}\right),
$$

with equality holds if and only if the hypertournament is regular.

For $k=2$ the degree sequence is identical to the score sequence given in [9]. In [27] Zhou and Zhang conjectured that a nondecreasing sequence $\mathrm{D}=\left[\mathrm{d}_{1}, \mathrm{~d}_{2}, \ldots, \mathrm{d}_{\mathrm{n}}\right]$ of nonnegative integers is a degree sequence of some $k$ hypertournament under some conditions, and proved for the case $k=3$.

The conjecture raised by Zhou and Zhang in [27] was settled by Chao and Zhou [24] and was obtained the following result.

Theorem 8 Given two positive integers $\mathrm{n}$ and $\mathrm{k}$ with $\mathrm{n}>\mathrm{k}>1$, a nondecreasing sequence $\left[\mathrm{d}_{1}, \mathrm{~d}_{2}, \ldots, \mathrm{d}_{n}\right]$ of nonnegative integers is a degree sequence of some k-hypertournament if and only if

$$
\sum_{i=1}^{r} d_{i} \geq\left(\begin{array}{l}
r \\
2
\end{array}\right)\left(\begin{array}{l}
n-2 \\
k-2
\end{array}\right),
$$

for all $1 \leq \mathrm{r} \leq \mathrm{n}$ with equality for $\mathrm{r}=\mathrm{n}$.

Let $H$ be a an $r$-uniform hypergraph with $r \geq 2$ and let $\alpha(H)$ be the vertex independence number of $\mathrm{H}$. In 2014 Chisthi, Zhou, Pirzada and Iványi [3] gave bounds for $\alpha(H)$ for different uniform hypergraphs.

\section{On stronger bounds in hypertournaments}

The following result is an equivalent form of Theorem 7 for scores and losing scores in a k-hypertournament. Here we give a different proof of this result. 
Theorem 9 Given two nonnegative integers $\mathrm{n}$ and $\mathrm{k}$ with $\mathrm{n} \geq \mathrm{k}>1$, if $\mathrm{S}=\left[\mathrm{s}_{1}, \mathrm{~s}_{2}, \ldots, \mathrm{s}_{\mathrm{n}}\right]$ of nonnegative integers in nonincreasing order is a score sequence and $\mathrm{R}=\left[\mathrm{r}_{1}, \mathrm{r}_{2}, \ldots, \mathrm{r}_{\mathrm{n}}\right]$ in non-decreasing order is the losing score sequence of some k-hypertournament, then

$$
\sum_{i=1}^{j} s_{i} r_{i} \leq\left(\begin{array}{l}
n \\
k
\end{array}\right)\left\{\left(\begin{array}{l}
n-1 \\
k-1
\end{array}\right)-\left(\begin{array}{l}
n \\
k
\end{array}\right) \frac{1}{n}\right\},
$$

with equality holds if and only if the hypertournament is regular.

Proof. Let $S=\left[s_{1}, s_{2}, \ldots, s_{n}\right]$ and $R=\left[r_{1}, r_{2}, \ldots, r_{n}\right]$ be respectively the score sequence and losing score sequence of a k-hypertournament $\mathrm{H}$, with $\mathrm{S}$ being non-increasing and $\mathrm{R}$ being nondecreasing. Then as a consequence of CauchySchwartz inequality, we have

$$
\left(\frac{r_{1}+r_{2}+\ldots+r_{n}}{n}\right)\left(\frac{s_{1}+s_{2}, \ldots+s_{n}}{n}\right) \geq \frac{r_{1} s_{1}+r_{2} s_{2}+\ldots+r_{n} s_{n}}{n}
$$

or

$$
\frac{1}{n} \sum_{i=1}^{n} s_{i} r_{i} \leq \frac{1}{n^{2}} \sum_{i=1}^{n} r_{i} \sum_{i=1}^{n} s_{i}
$$

or

$$
\sum_{i=1}^{n} s_{i} r_{i} \leq \frac{1}{n} \sum_{i=1}^{n} r_{i} \sum_{i=1}^{n} s_{i} .
$$

Now,

$$
\sum_{i=1}^{n}\left(s_{i}+r_{i}\right) r_{i}=\sum_{i=1}^{n} s_{i} r_{i}+\sum_{i=1}^{n} r_{i}^{2} .
$$

This gives,

$$
\sum_{i=1}^{n}\left(\begin{array}{l}
n-1 \\
k-1
\end{array}\right) r_{i}=\sum_{i=1}^{n} s_{i} r_{i}+\sum_{i=1}^{n} r_{i}^{2}
$$

(because $s_{i}+r_{i}=\left(\begin{array}{c}n-1 \\ k-1\end{array}\right), \quad 1 \leq i \leq n$ ).

So,

$$
\left(\begin{array}{l}
n-1 \\
k-1
\end{array}\right) \sum_{i=1}^{n} r_{i}=\sum_{i=1}^{n} s_{i} r_{i}+\sum_{i=1}^{n} r_{i}^{2},
$$

or

$$
\left(\begin{array}{l}
n-1 \\
k-1
\end{array}\right)\left(\begin{array}{l}
n \\
k
\end{array}\right)=\sum_{i=1}^{n} s_{i} r_{i}+\sum_{i=1}^{n} r_{i}^{2},
$$


(by the equality in Theorem 1).

Further, by the Chebyshev's inequality, we have,

$$
\sum_{i=1}^{n} s_{i} r_{i} \geq \frac{1}{n} \sum_{i=1}^{n} s_{i} \sum_{i=1}^{n} r_{i} .
$$

Using this in (6), we get

$$
\left(\begin{array}{l}
n-1 \\
k-1
\end{array}\right)\left(\begin{array}{l}
n \\
k
\end{array}\right) \geq \frac{1}{n} \sum_{i=1}^{n} s_{i} \sum_{i=1}^{n} r_{i}+\sum_{i=1}^{n} r_{i}^{2} .
$$

Since the arithmetic mean of $n$ non-negative real numbers never exceeds their root mean square, that is,

$$
\sqrt{\frac{\sum_{i=1}^{n} r_{i}^{2}}{n}} \geq \frac{\sum_{i=1}^{n} r_{i}}{n},
$$

with equality if and only if $r_{1}=r_{2}=\ldots=r_{n}$,

or

$$
\sum_{i=1}^{n} r_{i}^{2} \geq \frac{\left(\sum_{i=1}^{n} r_{i}\right)^{2}}{n}
$$

Using (8) in (7) we get

$$
\left(\begin{array}{l}
n-1 \\
k-1
\end{array}\right)\left(\begin{array}{l}
n \\
k
\end{array}\right) \geq \frac{1}{n} \sum_{i=1}^{n} s_{i} \sum_{i=1}^{n} r_{i}+\frac{\left(\sum_{i=1}^{n} r_{i}\right)^{2}}{n} .
$$

Therefore,

$$
\left(\begin{array}{l}
n-1 \\
k-1
\end{array}\right)\left(\begin{array}{l}
n \\
k
\end{array}\right) \geq \frac{1}{n} \sum_{i=1}^{n} s_{i} \sum_{i=1}^{n} r_{i}+\left(\begin{array}{l}
n \\
k
\end{array}\right)^{2} \frac{1}{n},
$$

or

$$
\begin{aligned}
\frac{1}{n} \sum_{i=1}^{n} s_{i} \sum_{i=1}^{n} r_{i} & \leq\left(\begin{array}{l}
n-1 \\
k-1
\end{array}\right)\left(\begin{array}{l}
n \\
k
\end{array}\right)-\frac{1}{n}\left(\begin{array}{l}
n \\
k
\end{array}\right)^{2} \\
& =\left(\begin{array}{l}
n \\
k
\end{array}\right)\left\{\left(\begin{array}{l}
n-1 \\
k-1
\end{array}\right)-\left(\begin{array}{l}
n \\
k
\end{array}\right) \frac{1}{n}\right\} .
\end{aligned}
$$

Using this in (5), we get

$$
\sum_{i=1}^{n} s_{i} r_{i} \leq\left(\begin{array}{l}
n \\
k
\end{array}\right)\left\{\left(\begin{array}{l}
n-1 \\
k-1
\end{array}\right)-\left(\begin{array}{l}
n \\
k
\end{array}\right) \frac{1}{n}\right\} .
$$


Now we show that the equality in (9) holds if and only if the hypertournament is regular, that is, if and only if $r_{1}=r_{2}=\ldots=r_{n}=r$.

Let $r_{1}=r_{2}=\ldots=r_{n}=r$ in $(9)$.

Then equality holds if and only if

$$
r \sum_{i=1}^{n} s_{i}=\left(\begin{array}{l}
n \\
k
\end{array}\right)\left\{\left(\begin{array}{l}
n-1 \\
k-1
\end{array}\right)-\left(\begin{array}{l}
n \\
k
\end{array}\right) \frac{1}{n}\right\} .
$$

That is, if and only if

$$
\frac{1}{n}\left(\begin{array}{l}
n \\
k
\end{array}\right) \sum_{i=1}^{n} s_{i}=\left(\begin{array}{l}
n \\
k
\end{array}\right)\left\{\left(\begin{array}{l}
n-1 \\
k-1
\end{array}\right)-\left(\begin{array}{l}
n \\
k
\end{array}\right) \frac{1}{n}\right\},
$$

(because by the equality in Theorem 1).

That is, if and only if

$$
\frac{1}{n}\left(\begin{array}{l}
n \\
k
\end{array}\right)\left\{n\left(\begin{array}{l}
n-1 \\
k-1
\end{array}\right)+0-\left(\begin{array}{l}
n \\
k
\end{array}\right)\right\}=\left(\begin{array}{l}
n \\
k
\end{array}\right)\left\{\left(\begin{array}{l}
n-1 \\
k-1
\end{array}\right)-\left(\begin{array}{l}
n \\
k
\end{array}\right) \frac{1}{n}\right\},
$$

(because by the equality in Theorem 2).

Therefore,

$$
\frac{1}{n}\left\{n\left(\begin{array}{l}
n-1 \\
k-1
\end{array}\right)-\left(\begin{array}{l}
n \\
k
\end{array}\right)\right\}=\left(\begin{array}{l}
n-1 \\
k-1
\end{array}\right)-\frac{1}{n}\left(\begin{array}{l}
n \\
k
\end{array}\right),
$$

or

$$
\left(\begin{array}{l}
n-1 \\
k-1
\end{array}\right)-\frac{1}{n}\left(\begin{array}{l}
n \\
k
\end{array}\right)=\left(\begin{array}{l}
n-1 \\
k-1
\end{array}\right)-\frac{1}{n}\left(\begin{array}{l}
n \\
k
\end{array}\right)
$$

which is true. Hence the equality in (9) holds, if and only if the hypertournament is regular.

The next result gives stronger bound for the total scores in k-hypertournaments.

Theorem 10 If $S=\left[s_{1}, s_{2}, \ldots, s_{n}\right]$ is a score sequence in non-decreasing order, $\mathrm{R}=\left[\mathrm{r}_{1}, \mathrm{r}_{2}, \ldots, \mathrm{r}_{\mathrm{n}}\right]$, is a losing score sequence in non-increasing order and $\mathrm{T}=\left[\mathrm{t}_{1}, \mathrm{t}_{2}, \ldots, \mathrm{t}_{\mathrm{n}}\right]$ is the total score sequence in non-increasing order of a $\mathrm{k}$-hypertournament $\mathrm{H}$, then for $1<\mathrm{p}<\infty$

$$
\sum_{i=1}^{l} t_{i}^{p} \geq l\left\{\left(\begin{array}{l}
n-1 \\
k-1
\end{array}\right)+\frac{2}{l}\left(\begin{array}{c}
n-l \\
k
\end{array}\right)-\frac{2}{l}\left(\begin{array}{l}
n \\
k
\end{array}\right)\right\}^{p},
$$

with equality holds if and only if $\mathrm{t}_{1}=\mathrm{t}_{2}=\ldots=\mathrm{t}_{\mathrm{n}}$ and $\mathrm{l}=\mathrm{n}$. 
Proof. Since the total score is $t_{i}=s_{i}-r_{i}$, then

$$
\sum_{i=1}^{l} t_{i}=\sum_{i=1}^{l} s_{i}-\sum_{i=1}^{l} r_{i}
$$

Therefore, by using Theorem 4 we obtain,

$$
\sum_{i=1}^{l} t_{i} \geq l\left(\begin{array}{l}
n-1 \\
k-1
\end{array}\right)+\left(\begin{array}{c}
n-l \\
k
\end{array}\right)-\left(\begin{array}{l}
n \\
k
\end{array}\right)-\left(\begin{array}{l}
n \\
k
\end{array}\right)+\left(\begin{array}{c}
n-l \\
k
\end{array}\right),
$$

or

$$
\sum_{i=1}^{l} t_{i} \geq l\left(\begin{array}{l}
n-1 \\
k-1
\end{array}\right)-2\left(\begin{array}{l}
n \\
k
\end{array}\right)+2\left(\begin{array}{c}
n-l \\
k
\end{array}\right) .
$$

But by the Holder's inequality with $\frac{1}{p}+\frac{1}{q}=1$ we have,

$$
\sum_{i=1}^{l} t_{i} \leq\left(\sum_{i=1}^{l} t_{i}^{p}\right)^{\frac{1}{p}}\left(\sum_{i=1}^{l} t_{i}^{q}\right)^{\frac{1}{q}}
$$

Using in (10), we get

$$
l \frac{1}{q}\left(\sum_{i=1}^{l} t_{i}^{p}\right)^{\frac{1}{p}} \geq l\left(\begin{array}{l}
n-1 \\
k-1
\end{array}\right)+2\left(\begin{array}{c}
n-l \\
k
\end{array}\right)-2\left(\begin{array}{l}
n \\
k
\end{array}\right),
$$

or

$$
\left(\sum_{i=1}^{l} t_{i}^{p}\right)^{\frac{1}{p}} \geq l^{-\frac{1}{q}}\left(l\left(\begin{array}{c}
n-1 \\
k-1
\end{array}\right)+2\left(\begin{array}{c}
n-l \\
k
\end{array}\right)-2\left(\begin{array}{l}
n \\
k
\end{array}\right)\right) .
$$

This implies

$$
\begin{aligned}
\left(\sum_{i=1}^{l} t_{i}^{p}\right)^{\frac{1}{p}} & \geq l^{-\frac{1}{q}} l\left(\begin{array}{c}
n-1 \\
k-1
\end{array}\right)+\frac{2}{l} l^{-\frac{1}{q}} l\left(\begin{array}{c}
n-l \\
k
\end{array}\right)-\frac{2}{l} l^{-\frac{1}{q}} l\left(\begin{array}{l}
n \\
k
\end{array}\right) \\
& \geq l^{1-\frac{1}{q}}\left(\begin{array}{c}
n-1 \\
k-1
\end{array}\right)+\frac{2}{l} l^{1-\frac{1}{q}}\left(\begin{array}{c}
n-l \\
k
\end{array}\right)-\frac{2}{l} l^{1-\frac{1}{q}}\left(\begin{array}{l}
n \\
k
\end{array}\right) \\
& =l^{1-\frac{1}{q}}\left(\left(\begin{array}{c}
n-1 \\
k-1
\end{array}\right)+\frac{2}{l}\left(\begin{array}{c}
n-l \\
k
\end{array}\right)-\frac{2}{l}\left(\begin{array}{l}
n \\
k
\end{array}\right)\right) \\
& =l^{\frac{1}{p}}\left(\left(\begin{array}{c}
n-1 \\
k-1
\end{array}\right)+\frac{2}{l}\left(\begin{array}{c}
n-l \\
k
\end{array}\right)-\frac{2}{l}\left(\begin{array}{l}
n \\
k
\end{array}\right)\right)
\end{aligned}
$$


(because $\frac{1}{\mathrm{p}}+\frac{1}{\mathrm{q}}=1$ or $\frac{1}{\mathrm{p}}=1-\frac{1}{\mathrm{q}}$ ),

which gives,

$$
\sum_{i=1}^{l} t_{i}^{p} \geq l\left(\left(\begin{array}{l}
n-1 \\
k-1
\end{array}\right)+\frac{2}{l}\left(\begin{array}{c}
n-l \\
k
\end{array}\right)-\frac{2}{l}\left(\begin{array}{l}
n \\
k
\end{array}\right)\right)^{p} .
$$

Now, we show that the equality in (11) holds, if and only if $t_{1}=t_{2}=\ldots=$ $t_{n}=t$ and $l=n$. That is, the equality in (11) holds if and only if

$$
n t^{p}=n\left(\left(\begin{array}{l}
n-1 \\
k-1
\end{array}\right)+\frac{2}{n}\left(\begin{array}{c}
n-n \\
k
\end{array}\right)-\frac{2}{n}\left(\begin{array}{l}
n \\
k
\end{array}\right)\right)^{p} .
$$

That is, if and only if

$$
t^{p}=\left(\left(\begin{array}{l}
n-1 \\
k-1
\end{array}\right)+\frac{2}{n}(0)-\frac{2}{n}\left(\begin{array}{l}
n \\
k
\end{array}\right)\right)^{p},
$$

or

$$
t=\left(\begin{array}{l}
n-1 \\
k-1
\end{array}\right)-\frac{2}{n}\left(\begin{array}{l}
n \\
k
\end{array}\right)
$$

or

$$
t=\frac{k}{n}\left(\begin{array}{l}
n \\
k
\end{array}\right)-\frac{2}{n}\left(\begin{array}{l}
n \\
k
\end{array}\right)=\frac{1}{n}\left(\begin{array}{l}
n \\
k
\end{array}\right)(k-2),
$$

which is the total score of a vertex in a regular k-hypertournament, because of the following fact.

By Theorem 5, we have for $l=n$, and $t_{1}=t_{2}=\ldots=t_{n}=t$

$$
\sum_{i=1}^{n} t=n\left(\begin{array}{l}
n-1 \\
k-1
\end{array}\right)-2\left(\begin{array}{l}
n \\
k
\end{array}\right) \text {. }
$$

This implies,

$$
n t=n\left(\begin{array}{l}
n-1 \\
k-1
\end{array}\right)-2\left(\begin{array}{l}
n \\
k
\end{array}\right)
$$

or

$$
t=\left(\begin{array}{l}
n-1 \\
k-1
\end{array}\right)-\frac{2}{n}\left(\begin{array}{l}
n \\
k
\end{array}\right) \text {. }
$$

Further, we can write

$$
t=\frac{k}{n}\left(\begin{array}{l}
n-1 \\
k-1
\end{array}\right)-\frac{2}{n}\left(\begin{array}{l}
n \\
k
\end{array}\right)=\frac{1}{n}\left(\begin{array}{l}
n \\
k
\end{array}\right)(k-2),
$$

which shows that, (12) and (13) are same. 


\section{Degrees in hypertournaments}

It is evident from Theorem 8 that inequalities on degrees play important role in the study of hypertournaments. We shall use the classical inequalities to provide more insight into the behavior of degrees in hypertournaments and hence the structure of hypertournaments. We also discuss the case of equality in detail for the inequalities derived and prove the equality holds if and only if the hypertournament is degree regular. We also obtain the necessary and sufficient conditions for the existence of a degree regular hypertournament.

Theorem 11 Let $\mathrm{n}$ and $\mathrm{k}$ be two positive integers with $\mathrm{n}>\mathrm{k}>1$. If $\mathrm{D}=$ $\left[\mathrm{d}_{1}, \mathrm{~d}_{2}, \ldots, \mathrm{d}_{\mathrm{n}}\right]$ is a degree sequence of some $\mathrm{k}$-hypertournament, then for a real number $\mathrm{p}$ with $1<\mathrm{p}<\infty$

$$
\sum_{i=1}^{r} d_{i}^{p} \geq \frac{r}{2^{p}}(r-1)^{p}\left(\begin{array}{l}
n-2 \\
k-2
\end{array}\right)^{p}
$$

where $1 \leq \mathrm{r} \leq \mathrm{n}$. In particular, for $\mathrm{r}=\mathrm{n}$

$$
\sum_{i=1}^{n} d_{i}^{p} \geq \frac{n}{2^{p}}(n-1)^{p}\left(\begin{array}{l}
n-2 \\
k-2
\end{array}\right)^{p},
$$

with equality in (15) holds if and only if the hypertournament is degree regular.

Proof. By Theorem 8, we have

$$
\sum_{i=1}^{r} d_{i} \geq\left(\begin{array}{l}
r \\
2
\end{array}\right)\left(\begin{array}{l}
n-2 \\
k-2
\end{array}\right),
$$

or

$$
\left(\begin{array}{l}
r \\
2
\end{array}\right)\left(\begin{array}{l}
n-2 \\
k-2
\end{array}\right) \leq \sum_{i=1}^{r} d_{i}
$$

But,

$$
\sum_{i=1}^{r} d_{i}=\sum_{i=1}^{r} d_{i} \cdot 1 \leq\left(\sum_{i=1}^{r} d_{i}^{p}\right)^{\frac{1}{p}}\left(\sum_{i=1}^{r} 1^{q}\right)^{\frac{1}{q}},
$$

(because by Holder's inequality with, $\frac{1}{p}+\frac{1}{q}=1$ ).

Hence,

$$
\left.\left(\begin{array}{l}
r \\
2
\end{array}\right)\left(\begin{array}{l}
n-2 \\
k-2
\end{array}\right) \leq\left(\sum_{i=1}^{r} d_{i}^{p}\right)\right)^{\frac{1}{p}}\left(\sum_{i=1}^{r} 1^{q}\right)^{\frac{1}{q}}
$$


(for, $1 \leq \mathrm{r} \leq \mathrm{n}$ and $\frac{1}{\mathrm{p}}+\frac{1}{\mathrm{q}}=1$ ).

That is,

$$
\left(\begin{array}{l}
r \\
2
\end{array}\right)\left(\begin{array}{l}
n-2 \\
k-2
\end{array}\right) \leq\left(\sum_{i=1}^{r} d_{i}^{p}\right)^{\frac{1}{p}} r^{\frac{1}{q}},
$$

or

$$
r^{-\frac{1}{q}}\left(\begin{array}{l}
r \\
2
\end{array}\right)\left(\begin{array}{l}
n-2 \\
k-2
\end{array}\right) \leq\left(\sum_{i=1}^{r} d_{i}^{p}\right)^{\frac{1}{p}},
$$

or

$$
\frac{r^{-\frac{1}{q}} r(r-1)(r-2) !}{2(r-2) !\left(\begin{array}{l}
n-2 \\
k-2
\end{array}\right)} \leq\left(\sum_{i=1}^{r} d_{i}^{p}\right)^{\frac{1}{p}}
$$

or

$$
r^{1-\frac{1}{q}} \frac{(r-1)}{2}\left(\begin{array}{l}
n-2 \\
k-2
\end{array}\right) \leq\left(\sum_{i=1}^{r} d_{i}^{p}\right)^{\frac{1}{p}}
$$

This gives,

$$
r^{\frac{1}{p}} \frac{(r-1)}{2}\left(\begin{array}{l}
n-2 \\
k-2
\end{array}\right) \leq\left(\sum_{i=1}^{r} d_{i}^{p}\right)^{\frac{1}{p}}
$$

(because $\frac{1}{p}+\frac{1}{q}=1$ or $\frac{1}{p}=1-\frac{1}{q}$ ).

Hence,

$$
\sum_{i=1}^{r} d_{i}^{p} \geq \frac{r(r-1)^{p}}{2^{p}}\left(\begin{array}{l}
n-2 \\
k-2
\end{array}\right)^{p} .
$$

For $r=\mathfrak{n}$, we have by the equality in Theorem 8

$$
\sum_{i=1}^{n} d_{i}=\left(\begin{array}{l}
n \\
2
\end{array}\right)\left(\begin{array}{l}
n-2 \\
k-2
\end{array}\right) .
$$

So, inequality (16) now becomes

$$
\sum_{i=1}^{n} d_{i}^{p} \geq \frac{n}{2^{p}}(n-1)^{p}\left(\begin{array}{l}
n-2 \\
k-2
\end{array}\right)^{p} .
$$

Further, we show that the equality in (17) holds if and only if $d_{1}=d_{2}=$ $\ldots=d_{n}=d$, that is, if and only if the hypertournament is degree regular.

Suppose $d_{1}=d_{2}=\ldots=d_{n}=d$ in (17). Then equality holds if and only if

$$
\sum_{i=1}^{n} d_{i}^{p}=\frac{n}{2^{p}}(n-1)^{p}\left(\begin{array}{l}
n-2 \\
k-2
\end{array}\right)^{p} .
$$


That is, if and only if

$$
n d^{p}=\frac{n}{2^{p}}(n-1)^{p}\left(\begin{array}{l}
n-2 \\
k-2
\end{array}\right)^{p}
$$

or

$$
d^{p}=\frac{(n-1)^{p}}{2^{p}}\left(\begin{array}{l}
n-2 \\
k-2
\end{array}\right)^{p}
$$

That is, if and only if

$$
d=\frac{(n-1)}{2}\left(\begin{array}{l}
n-2 \\
k-2
\end{array}\right)
$$

which clearly is the degree of a vertex in a regular k-hypertournament, verified as follows. We know by the equality in Theorem 8

$$
\sum_{i=1}^{n} d_{i}=\left(\begin{array}{l}
n \\
2
\end{array}\right)\left(\begin{array}{l}
n-2 \\
k-2
\end{array}\right) .
$$

Since $d_{1}=d_{2}=\ldots=d_{n}=d$.

$$
n d=\left(\begin{array}{l}
n \\
2
\end{array}\right)\left(\begin{array}{l}
n-2 \\
k-2
\end{array}\right)
$$

which gives,

or

$$
n d=\frac{n(n-1) !}{2(n-2) !}\left(\begin{array}{l}
n-2 \\
k-2
\end{array}\right)
$$

$$
d=\frac{(n-1)}{2}\left(\begin{array}{l}
n-2 \\
k-2
\end{array}\right) \text {. }
$$

Clearly (18) is same as (19).

The following result gives the conditions for the existence of a degree regular k-hypertournament on $n$ vertices.

Theorem 12 Let $\mathrm{n}$ and $\mathrm{k}$ be two positive integers. For $\mathrm{n}>2$ and $\mathrm{n}>\mathrm{k}>1$, there exists a degree regular $k$-hypertournament on $\mathrm{n}$ vertices if and only if $\mathrm{n}$ divides $\left(\begin{array}{l}k \\ 2\end{array}\right)\left(\begin{array}{l}n \\ k\end{array}\right)$.

Proof. Suppose there exists a degree regular k-hypertournament with its degree sequence $\left[d_{1}, d_{2}, \cdots, d_{n}\right]$. Then by the inequality (16), we have

$$
\sum_{i=1}^{n} d^{2}=\frac{n}{2^{2}}(n-1)^{2}\left(\begin{array}{l}
n-2 \\
k-2
\end{array}\right)^{2},
$$


(case of equality with $p=2$, where $d_{1}=d_{2}=\ldots=d_{n}=d$ ).

Thus,

$$
n d^{2}=\frac{n}{2^{2}}(n-1)^{2}\left(\begin{array}{l}
n-2 \\
k-2
\end{array}\right)^{2}
$$

or

$$
d^{2}=\frac{(n-1)^{2}}{2^{2}}\left(\begin{array}{l}
n-2 \\
k-2
\end{array}\right)^{2}
$$

This gives,

$$
d=\frac{(n-1)}{2}\left(\begin{array}{l}
n-2 \\
k-2
\end{array}\right)
$$

(because degree cannot be negative).

Now,

$$
2 d=(n-1)\left(\begin{array}{l}
n-2 \\
k-2
\end{array}\right)
$$

or

$$
\frac{2 d}{k(k-1)}=\frac{(n-1)(n-2) !}{k(k-1)(k-2) !(n-k) !}
$$

or

$$
2 n d=\frac{k(k-1) n !}{k !(n-k) !}
$$

or

$$
n d=\frac{k(k-1)}{2}\left(\begin{array}{l}
n \\
2
\end{array}\right)
$$

Conversely, suppose that $n$ divides $\left(\begin{array}{l}k \\ 2\end{array}\right)\left(\begin{array}{l}n \\ k\end{array}\right)$.

Set for each $1 \leq i \leq n$,

$$
d_{i}=\frac{1}{n}\left(\begin{array}{l}
k \\
2
\end{array}\right)\left(\begin{array}{l}
n \\
k
\end{array}\right)=\frac{k(k-1)}{2 n}\left(\begin{array}{l}
n \\
k
\end{array}\right) .
$$

Then,

$$
\begin{aligned}
d_{i} & =\frac{k(k-1)}{2 n} \frac{n !}{k !(n-k) !} \\
& =\frac{k(k-1) n(n-1)(n-2) !}{2 n k(k-1)(k-2) !(n-k) !} \\
& =\frac{(n-1)}{2}\left(\begin{array}{l}
n-2 \\
k-2
\end{array}\right) .
\end{aligned}
$$


Therefore,

$$
\sum_{i=1}^{r} d_{i}=\sum_{i=1}^{r}\left\{\frac{n-1}{2}\left(\begin{array}{l}
n-2 \\
k-2
\end{array}\right)\right\},
$$

which implies, for $1 \leq \mathrm{r} \leq \mathrm{n}$

$$
\begin{aligned}
\sum_{i=1}^{r} d_{i} & =\frac{r(n-1)}{2}\left(\begin{array}{l}
n-2 \\
k-2
\end{array}\right) \\
& \geq \frac{r(r-1)}{2}\left(\begin{array}{l}
n-2 \\
k-2
\end{array}\right) \\
& =\frac{r(r-1)(r-2) !}{2(r-2) !}\left(\begin{array}{l}
n-2 \\
k-2
\end{array}\right),
\end{aligned}
$$

(because $n \geq r$ implies $(n-1) \geq(r-1))$.

Hence,

$$
\sum_{i=1}^{r} d_{i} \geq\left(\begin{array}{l}
r \\
2
\end{array}\right)\left(\begin{array}{l}
n-2 \\
k-2
\end{array}\right),
$$

with equality when $r=n$.

Thus by Theorem $8, D=\left[d_{1}, d_{2}, \ldots, d_{n}\right]$ is the degree sequence of a degree regular k-hypertournament.

\section{References}

[1] R. Assous, Enchainabilite et seuil de monomorphie des tournois n-aires, Discrete Math. 62 (1986), 119-125. $\Rightarrow 201$

[2] E. Barbut, A. Bialostocki, A generalization of rotational tournaments, Discrete Math. 76 (1989), 81-87. $\Rightarrow 201$

[3] T. A. Chisthi, G. Zhou, S. Pirzada, A. Iványi, On independence numbers of uniform hypergraphs, Acta Univ. Sapientiae, Informatica 6, 1 (2014) 132-158. $\Rightarrow 204$

[4] P. Frankl, What must be contained in every oriented k-uniform hypergraph, Discrete Math. 62 (1986), 311-313. $\Rightarrow 201$

[5] G. Gutin, A. Yeo, Hamiltonian paths and cycles in hypertournaments, J. Graph Theory 25 (1997), 277-286. $\Rightarrow 201$

[6] K. K. Kayibi, M. A. Khan, S. Pirzada, Uniform sampling of k-hypertournaments, Linear and Multilinear Algebra 61, 1 (2013), 123-138. $\Rightarrow 201$

[7] Y. Koh, S. Ree, On k-hypertournament matrices, Lin. Alg. Appl. 373 (2002) 183-195. $\Rightarrow 202,203$ 
[8] M. A. Khan, S. Pirzada, K. K. Kayibii, Scores, Inequalities and regular hypertournaments, J. Math. Inequal. Appl. 15, 2 (2012) 343-351. $\Rightarrow 203,204$

[9] H. G. Landau, On dominance relations and the structure of animal societies, III, The condition for a score structure, Bull. Math. Biophys. 15 (1953) 143-148. $\Rightarrow 201,204$

[10] S. Pirzada, On scores in multipartite hypertournaments, Eurasian Math. Journal, 2, 1 (2011) 112-119. $\Rightarrow 201$

[11] S. Pirzada, An Introduction to Graph Theory, Universities Press, Orient Blackswan, 2012. $\Rightarrow 201$

[12] S. Pirzada, Score lists in bipartite multi hypertournaments, Math. Vesnik 64, 4 (2012) 286-296. $\Rightarrow 201$

[13] S. Pirzada, hypertournaments-Scores, losing scores, total scores and degrees, $J$. Comp. Math. Comb. Comp. 84 (2013) 95-108. $\Rightarrow 201$

[14] S. Pirzada, T. A. Naikoo, Score sets in tournaments, Vietnam J. Math. 34, 2 (2006) 157-161. $\Rightarrow 201$

[15] S. Pirzada, U. Samee, Mark sequences in digraphs, Seminaire Lotharingien de Combinatoire 55 (2006) B55c. $\Rightarrow 201$

[16] S. Pirzada, T. A. Naikoo, N. A. Shah, Score sequences in oriented graphs, J. Appl. Math. Comp. 23, 1-2 (2007) 257-268. $\Rightarrow 201$

[17] S. Pirzada, G. Zhou, Score lists in (h,k)-bipartite hypertournaments, Appl. Math. J. Chinese Universities, Series B 22, 4 (2007) 485-489. $\Rightarrow 201$

[18] S. Pirzada,, T. A. Naikoo, G. Zhou, Score lists in tripartite hypertournaments, Graphs and Combinatorics 23, 4 (2007) 445-454. $\Rightarrow 201$

[19] S. Pirzada, T. A. Naikoo, Score sets in oriented graphs, Appl. Analysis and Discrete Math. 2, 1 (2008) 107-113. $\Rightarrow 201$

[20] S. Pirzada, G. Zhou, On k-hypertournament losing scores, Acta Univ. Sapientiae, Informatica 2, 1 (2010) 5-9. $\Rightarrow 201$

[21] S. Pirzada, G. Zhou, A. Iványi, Score lists in multipartite hypertournaments, Acta Univ. Sapientiae, Informatica 2, 2 (2010) 184-193. $\Rightarrow 201$

[22] S. Pirzada, G. Zhou, Degree lists in k-bipartite hypertournaments, TWMS J. Pure and Appl. Math. 5, 2 (2014), 89-116. $\Rightarrow 201$

[23] S. Pirzada, M. A. Khan, G. Zhou, K. K. Kayibi, On scores, losing scores and total scores in hypertournaments, Electronic J. Graph Theory Appl. 3, 1 (2015) $8-21 . \Rightarrow 201$

[24] C. Wang, G. Zhou, Note on the degree sequences of k-hypertournaments, Discrete Math. 308, 11 (2008) 2292-2296. $\Rightarrow 204$

[25] G. Zhou, S. Pirzada, Degree sequences in oriented k-hypergraphs, J. Appl. Math., Comp. 27, 1-2 (2008) 149-158. $\Rightarrow 201$

[26] G. Zhou, Y. Tianxing, Z. Kemin, On score sequences of k-hypertournament, Europ. J. Comb. 21 (2000), 993-1000. $\Rightarrow 201$

[27] G. Zhou, K. Zhang, On the degree sequences of k-hypertournaments, Chinese Annals of Math., Series A 22, 1 (2001) 115-120. $\Rightarrow 204$

Received: November 4, 2015 • Revised: December 22, 2015 